Inc., Employee of: Myriad Genetics, Inc., Megan Horton Shareholder of: Myriad Genetics, Inc., Employee of: Myriad Genetics, Inc., Darl Flake Shareholder of: Myriad Genetics, Inc., Employee of: Myriad Genetics, Inc., Eric Sasso Shareholder of: Myriad Genetics, Inc., Employee of: Myriad Genetics, Inc., Michael E. Weinblatt Grant/research support from: BMS, Amgen, Lilly, Crescendo and Sonofi-Regeneron, Consultant of: Horizon Therapeutics, Bristol-Myers Squibb, Amgen, Abbvie, Crescendo, Lilly, Pfizer, Roche, Gilead DOI: 10.1136/annrheumdis-2020-eular.1188

\section{AB1240 C4B BINDING PROTEIN AND WARFARIN IN THE ANTIPHOSPHOLIPID SYNDROME}

G. Grosso ${ }^{1}$, K. Sandholm ${ }^{2}$, I. Gunnarsson ${ }^{1}$, A. Zickert ${ }^{1}$, A. Vikerfors ${ }^{3}$, A. Antovic ${ }^{1}$, B. Nilsson ${ }^{4}$, K. Nilsson Ekdahl ${ }^{2,4}$, E. Svenungsson ${ }^{1} .{ }^{1}$ Karolinska University, Rheumatology, Solna, Sweden; ${ }^{2}$ Linnaeus University, Center for Biomaterials Chemistry, Kalmar, Sweden; ${ }^{3}$ Swedish Medical Products Agency, Uppsala, Sweden; ${ }^{4}$ Uppsala University, Immunology, Genetics and Pathology, Uppsala, Sweden

Background: Complement plays a role in the Antiphospholipid Syndrome (APS). C4b Binding Protein (C4BP) is a complement inhibitor with anticoagulant function (1). It belongs to the same protein family as $\beta 2 G P I$, the main antigen in APS. Its main isoform is bound to protein $S$ in the circulation. Levels of both protein $S$ and C4BP are known to be reduced by warfarin treatment (2) as well as by aPL, directly and indirectly.

Objectives: To investigate the levels of C4BP in primary ( $p$ ) and secondary (s) APS, also considering warfarin treatment.

Methods: The total amount of C4BP (C4BPt) was measured by using magnetic carboxylated microspheres which were coupled with a monoclonal antibody against the a-chain of human-C4BP to capture the antigen. To detect C4BPt the same antibody was used, biotinylated. The binding of biotinylated antibodies was detected by streptavidin-phycoerythrin and data were collected using a MAGPIX Multiplex Reader. Using independent t-test, we compared C4BP in 118 SLE patients with repeated positivity for Antiphospholipid antibodies (aPL) (39/118 on warfarin), $291 \mathrm{aPL}$ negative SLE patients (16/291 on warfarin), 67 pAPS (33/67 on warfarin), and 322 controls (none on warfarin). We then performed an interaction and a mediation analysis (3) in the SLE group to study the impact of warfarin on C4BP levels: since warfarin is mostly prescribed to $\mathrm{aPL}+$ patients, it is considered a mediator in the reducing effect of aPL on C4BP. Therefore we compared individuals exposed and non-exposed to the presence of aPL with or without the mediator warfarin and calculated the percentage of reduction in C4BP that could be attributed to $\mathrm{aPL}$ or warfarin.

Results: Overall C4BP is $20 \%$ reduced in aPL+ patients (fig 1), independently of SLE, past thrombotic events and nephritis. Warfarin treated patients have lower levels of C4BP (fig 2). According to mediation analysis $11 \%$ of $\mathrm{C} 4 \mathrm{BP}$ reduction is due to $\mathrm{aPL}$ and $9 \%$ to warfarin.

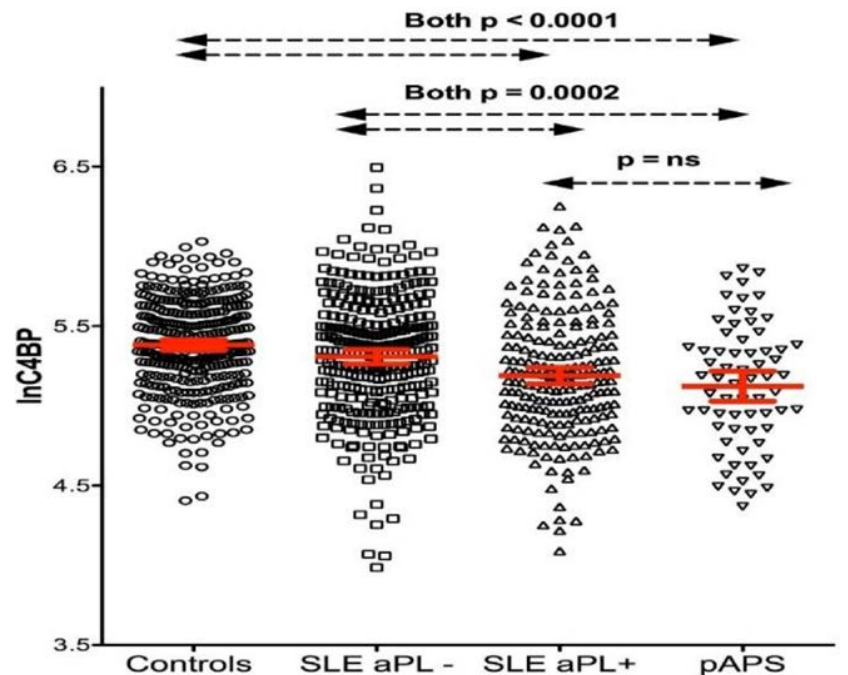

Figure 1. C4BP in different subgroups (67 pAPS, 118 SLEaPL+, 291 SLEaPL-, 322 controls)

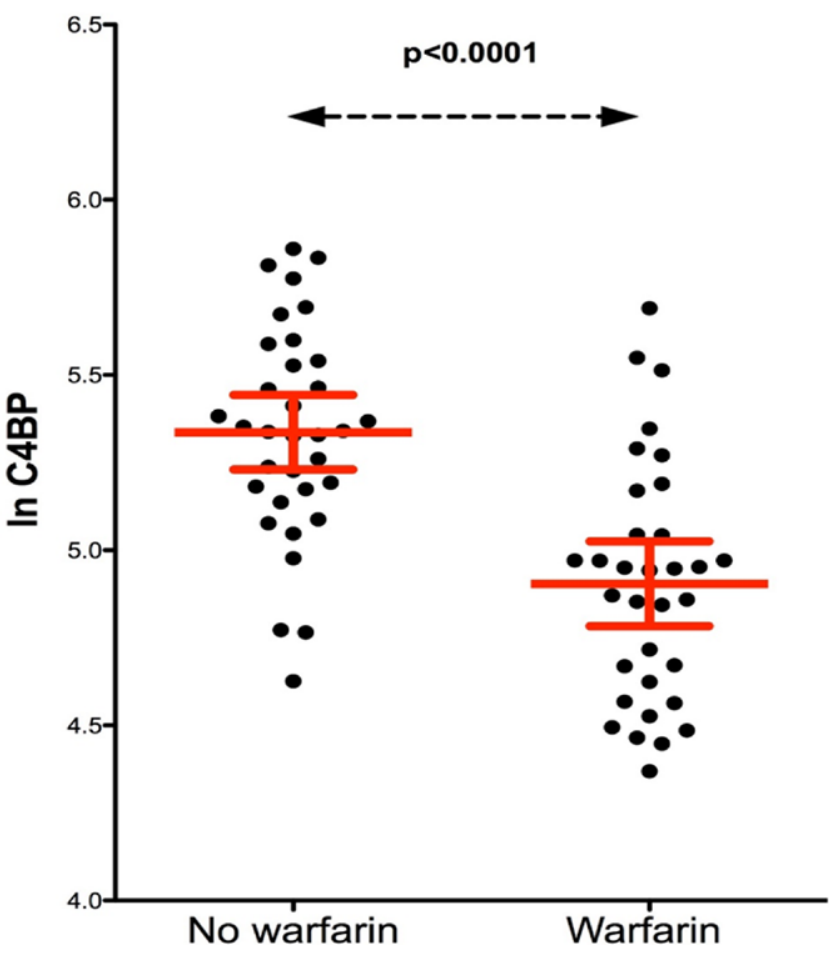

Figure 2. C4BP in 67 pAPS patients, $33 / 67$ on warfarin

Conclusion: Both aPL and warfarin decrease levels of C4BP, a complement and coagulation regulator. Reduction of this complement inhibitor could contribute to complement activation and thrombosis in APS. Our results raise new questions regarding the effects of warfarin treatment on complement and coagulation in APS.

References: [1]Dahlbäck B. C4b-binding protein: a forgotten factor in thrombosis and hemostasis. Seminars in thrombosis and hemostasis 2011; 37(4): 355.

[2] Zöller B, García de Frutos P, Dahlbäck B. Evaluation of the relationship between protein $\mathrm{S}$ and $\mathrm{C} 4 \mathrm{~b}$-binding protein isoforms in hereditary protein $S$ deficiency demonstrating type I and type III deficiencies to be phenotypic variants of the same genetic disease. Blood 1995; 85(12): 3524

[3] Vanderweele TJ, Vansteelandt S. Conceptual issues concerning mediation, interventions and composition. Statistics and Its Interface 2009; 2(4): 457-68. Disclaimer: AV is employed at the Swedish Medical Products Agency, the views expressed in this paper are the personal views of the authors and not necessarily the views of the Governement Agency

Acknowledgments: Thanks to the Biostatistics Core Facility, Karolinska University

Disclosure of Interests: None declared

DOI: 10.1136/annrheumdis-2020-eular.5606

\section{AB1241 1 EVALUATION OF A PATIENT COMPLETED DISEASE FLARE QUESTIONNAIRE IN PSORIATIC DISEASE}

P. Helliwell ${ }^{1}$, W. Tillett ${ }^{2}$, R. Waxman ${ }^{1}$, L. C. Coates ${ }^{3}$, O. Fitzgerald ${ }^{4}$, J. Packham ${ }^{5}$ $\mathrm{N}$. Mchugh ${ }^{6}$ on behalf of On behalf of the PROMPT study group. ${ }^{1}$ Chapel Allerton Hospital, Leeds, United Kingdom; ${ }^{2}$ Royal National Hospital for Rheumatic Diseases, Bath, United Kingdom; ${ }^{3}$ University of Oxford, Oxford, United Kingdom; ${ }^{4}$ University College Dublin, Dublin, Ireland; ${ }^{5}$ University of Nottingham, Nottingham, United Kingdom; ${ }^{6}$ University of Bath, Bath, United Kingdom

Background: Psoriatic Disease (PsD) is a chronic inflammatory disease of the skin, nails, joints, and entheses. A number of composite disease activity measures have been developed though there is yet consensus as to which to use in the clinic and in clinical trials. A patient completed disease flare questionnaire, covering multiple domains of disease impact, has been developed but has yet to be fully validated.

Objectives: To validate the FLARE questionnaire in PsD.

Methods: The 10 question FLARE instrument ${ }^{1}$ was administered to 141 patients in an observational study of treatment change in PsD over 6 months follow up. Disease activity was measured by the PASDAS and the gold standard of flare 